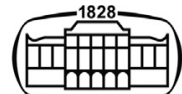

AKADÉMIAI KIADÓ

Journal of Behavioral Addictions

10 (2021) 3, 540-545

Special Section on

Ovenweight, obesity and eating disorders in relation to addiction

\section{DOI:}

$10.1556 / 2006.2021 .00060$

(c) 2021 The Author(s)

\section{BRIEF REPORT}

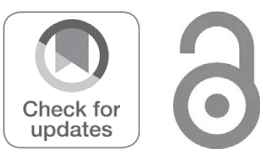

\title{
Eating disorder in gambling disorder: A group with increased psychopathology
}

\section{LINDA LEMÓN ${ }^{1}$, FERNANDO FERNÁNDEZ-ARANDA ${ }^{2,3,4} \oplus$, SUSANA JIMÉNEZ-MURCIA ${ }^{2,3,4} \odot$ and ANDERS HÅKANSSON ${ }^{5,6 *}$ (1)}

\author{
${ }^{1}$ Region Skåne, Department of Psychiatry Malmö-Trelleborg, Malmö, Sweden \\ ${ }^{2}$ Department of Psychiatry, Bellvitge University Hospital-IDIBELL, Barcelona, Spain \\ ${ }^{3}$ Ciber Fisiopatología Obesidad y Nutrición (CIBERObn), Instituto de Salud Carlos III, Madrid, Spain \\ ${ }^{4}$ Department of Clinical Sciences, School of Medicine and Health Sciences, University of Barcelona, \\ Barcelona, Spain \\ ${ }^{5}$ Region Skåne, Malmö Addiction Center, Malmö, Sweden \\ ${ }^{6}$ Lund University, Faculty of Medicine, Department of Clinical Sciences Lund, Psychiatry, Lund, Sweden
}

Received: May 12, 2021 • Revised manuscript received: June 29, 2021 • Accepted: August 14, 2021

Published online: September 28, 2021

\begin{abstract}
Background and aims: Theoretical background and previous data provide some similarities between problematic gambling and eating behaviors, and a theoretically increased clinical severity in individuals suffering from both conditions. However, large datasets are lacking, and therefore, the present study aimed to study, in a nationwide register material, psychiatric comorbidity, age and gender in gambling disorder (GD) patients with or without eating disorder (ED). Methods: Diagnostic data from a nationwide register were used, including all individuals with a GD diagnosis in specialized health care in Sweden, in the years 2005-2016 $(N=2,099)$. Patients with GD and an ED diagnosis $(n=57)$ were compared to GD patients without ED. Results: Patients with GD+ED were significantly more likely than other GD patients to also have a diagnosis of drug use disorder, depressive disorders, bipolar disorders, other mood disorder, anxiety disorders, personality disorders, and neuropsychiatric disorders, when controlling for gender. In logistic regression, a comorbid ED in GD was associated with female gender, younger age, depressive disorder and personality disorders. Discussion and conclusion: In nationwide register data, despite the low number of GD+ED patients, GD patients with ED appear to have a more severe psychiatric comorbidity than GD patients without $\mathrm{ED}$. The combined GD+ED conditions may require particular screening and clinical attention, as well as further research in larger and longitudinal studies.
\end{abstract}

\section{KEYWORDS}

eating disorder, gambling disorder, comorbidity, behavioral addiction

\section{INTRODUCTION}

Problem gambling is common, with a past-year prevalence of $0.1-5.8 \%$ of the population (Calado and Griffiths, 2016), and gambling disorder (GD) is associated with severe financial and social consequences (Langham et al., 2016) and suicidal behavior (Karlsson \& Håkansson, 2018). Eating disorders (EDs) have an estimated lifetime prevalence at around $1 \%$; around $0.2 \%$ for anorexia nervosa $(\mathrm{AN}), 0.8 \%$ bulimia nervosa $(\mathrm{BN})$, and $2.2 \%$ binge eating disorder (BED), and are 4.2 times more common among women than among men (Qian et al., 2013). EDs are associated with severely decreased quality of life, particularly in patients with binge/purge diagnoses (DeJong et al., 2013), and different addictions are associated with ED (Bahji et al., 2019); patients with ED have higher lifetime and current drug use (Krug et al., 2008). BN and AN-binge-purging (AN-BP) generally present the highest rates, and $\mathrm{AN}$-restrictive $(\mathrm{AN}-\mathrm{R})$ the lowest rates. 
Several studies have demonstrated associations between GD and ED. ED, especially the binge/purge type characterized by high impulsivity, are common in treatment (Jimenez-Murcia et al., 2013) and community samples (von Ranson, Wallace, Holub, \& Hodgins, 2013) of patients with GD. Problematic gambling is elevated among people with BN (Fernandez-Aranda et al., 2006) and BED (Yip, White, Grilo, \& Potenza, 2011). von Ranson et al. (2013) found a high rate of current ED psychopathology among 38\% of female vs $4 \%$ of male community-recruited adults with problem gambling.

Binge eating may be conceptualized as a behavioral addiction similar to GD (Farstad et al., 2015). Addictionrelated eating behavior is typically referred to as food addiction (FA) (Gearhardt, Corbin, \& Brownell, 2009), with a high prevalence in ED patients, possibly up to $87 \%$ (Romero et al., 2019). Like ED, FA is more common in women (Pursey, Stanwell, Gearhardt, Collins, \& Burrows, 2014) and in binge disorders (Jiménez-Murcia, Agüera, Paslakis, 2019). In patients with GD, the prevalence of FA in one study was found to be $31 \%$ in women and $7 \%$ in men (Jimenez-Murcia et al., 2017). A growing body of evidence suggests that BED shares similarities with conventional addictions, and that some forms of excessive overeating might be more appropriately described as an addiction disorder like FA (Davis et al., 2011; Jiménez-Murcia et al., 2019).

Altogether, there are indications of GD and ED belonging to a similar or overlapping disease spectrum, and deeper understanding of this may advise clinicians and stakeholders to improve cross-diagnostic screening (Jimenez-Murcia et al., 2013). For example, if the group with both GD and ED would present with more pronounced clinical problems, this would be a reason to improve structured screening for the combined condition. Still, few studies have analyzed the comorbidity between GD and ED, and larger samples, such as nationwide samples, are lacking. This might be explained by the fact that GD is more frequent in males whereas ED are more frequent in women. The purpose of this study is to investigate, in nationwide Swedish register data, the relationship between GD and the diagnostic group of $\mathrm{ED}$, and to examine whether individuals with both GD and ED have higher psychiatric comorbidity than individuals with GD without ED.

\section{METHODS}

The present study is a register-based, nationwide study of patients who have received a GD diagnosis in specialized health care in Sweden, a country with problem gambling rates in the mid-range of comparable European countries and within the range of other Scandinavian countries (Calado \& Griffiths, 2016). The study uses the Swedish patient register, which includes diagnoses according to the ICD-10 for patients diagnosed in in-patient hospital care or specialized out-patient care, i.e. all out-patient care except primary care ('GP') facilities. As the treatment uptake for GD traditionally has been low in Swedish health care, it can be assumed that a minority of individuals with a GD appear in the patient register, and it can be suspected that this cohort is skewed towards a higher problem level than the general population of GD populations, although such comparisons cannot readily be made from available data (Karlsson \& Hakansson, 2018). Available data used in the study include all psychiatric diagnoses occurring at any time during the study period (years 2005-2016, categorized into diagnostic categories), as well as gender and age.

The sample used here comprises all individuals with a gambling disorder diagnosis (ICD-10 code F63.0) in the patient register during 2005-2016, and is the same cohort as previously used in nationwide research on GD and mortality. As in previous analyses, the limited number of individuals below 18 years of age were excluded. Legal gambling age is 18 years, and given the close linguistic association between the terms 'gambling' and 'gaming' in Swedish language, and the limited role of these conditions in mental health facilities traditionally, it was considered hard to exclude that younger individuals with a predominantly gaming-oriented problem could have been mistakenly classified with a F63.0 diagnosis (Karlsson \& Håkansson, 2018).

A total of 2,099 individuals with a GD diagnosis were included ( $23 \%$ women, $n=474)$, with a mean age of 36.5 years.

\section{Statistical methods}

The study is a statistical comparison of patients with GD and any occurrence of an eating disorder of any sub-type $(\mathrm{GD}+\mathrm{ED})$ during the study period, and patients with GD without an ED. Groups were compared with respect to age, gender, and occurrence of other diagnostic categories during the study period; alcohol use disorders (F10), drug use disorders (F11-19), misuse of non-addictive drugs (F55), psychotic disorders (F2), depressive disorders, bipolar disorders, other mood disorders, anxiety and stress-related disorders (F4), personality disorders, impulse control disorders (other than gambling disorder, F63.0), sexual disorders, and neuropsychiatric disorders.

Group comparisons were made using the chi-square test for categorical variables and $t$-test for age. Given the strong association between ED prevalence and gender, all other variables were controlled for gender, one by one. Variables with a significant association with the GD+ED group (after adjusting for gender) were entered simultaneously in a logistic regression analysis, including gender and age, with the $\mathrm{GD}+\mathrm{ED}$ status as the dependent variable. Analyses were carried out in the IBM SPSS software, version 25.0. The level of significance used was $P<0.05$. Odds ratios were reported with $95 \%$ confidence intervals.

\section{Ethics}

This overall project of comorbidity in gambling disorder was approved by the Regional Ethics Board, Lund, Sweden (file number 2016/1104). No informed consent procedure was required for the present study, in line with the decision of 
Table 1. Characteristics of patients with GD with or without ED

\begin{tabular}{|c|c|c|c|c|}
\hline & $\mathrm{GD}+\mathrm{ED}(n=57), \%(\mathrm{n})$ & GD-ED $(n=2,042), \%(\mathrm{n})$ & $P$ value & Adjusted for gender \\
\hline Female gender & $68 \%(39)$ & $21 \%(435)$ & $<0.001$ & - \\
\hline Age, mean & 32.2 & 36.6 & 0.005 & \\
\hline Alcohol use disorders & $35 \%(20)$ & $29 \%(598)$ & 0.34 & 0.298 \\
\hline Drug use disorders & $47 \%(27)$ & $25 \%(507)$ & $<0.001$ & $<0.001$ \\
\hline $\begin{array}{l}\text { Substance misuse, non-addictive } \\
\text { substances (F55) }\end{array}$ & $0 \%(0)$ & $0 \%(9)$ & 1.00 & * \\
\hline Psychotic disorder & $9 \%(5)$ & 9\% (191) & 0.88 & * \\
\hline Depressive disorder & $81 \%(46)$ & $50 \%(1,027)$ & $<0.001$ & 0.001 \\
\hline Bipolar disorder & $26 \%(15)$ & $12 \%(235)$ & 0.001 & 0.023 \\
\hline Other mood disorder & $18 \%(10)$ & $6 \%(113)$ & $<0.001$ & 0.004 \\
\hline Anxiety disorder & $84 \%(48)$ & $59 \%(1,204)$ & $<0.001$ & 0.003 \\
\hline Personality disorder & $54 \%(31)$ & $18 \%(377)$ & $<0.001$ & $<0.001$ \\
\hline - Emotionally unstable & $40 \%(23)$ & $9 \%(186)$ & $<0.001$ & $<0.001$ \\
\hline - Histrionic & $4 \%(2)$ & $0 \%(3)$ & $<0.01^{\star \star}$ & * \\
\hline - Paranoid & $4 \%(2)$ & $2 \%(32)$ & 0.24 & * \\
\hline - Schizoid & $2 \%(1)$ & $0 \%(10)$ & 0.26 & * \\
\hline - Antisocial & $4 \%(2)$ & $3 \%(53)$ & 0.66 & * \\
\hline - Anankastic & $0 \%(0)$ & $0 \%(3)$ & 1.00 & * \\
\hline - Anxious & $4 \%(2)$ & $1 \%(20)$ & 0.12 & * \\
\hline - Dependent & $0 \%(0)$ & $1 \%(19)$ & 1.00 & * \\
\hline - Other & $30 \%(17)$ & $10 \%(201)$ & $<0.001$ & $<0.001$ \\
\hline Impulse control disorder & $4 \%(2)$ & $5 \%(97)$ & 1.00 & * \\
\hline Sexual disorder & $2 \%(1)$ & $1 \%(27)$ & $0.54^{* *}$ & * \\
\hline Neuropsychiatric disorder & $30 \%(17)$ & $17 \%(343)$ & 0.01 & 0.014 \\
\hline
\end{tabular}

${ }^{\star}$ Variables with low absolute numbers were not run in logistic regression.

${ }^{*}$ Fisher's exact test.

the ethics board, as the study did not contain data on identified individuals.

\section{RESULTS}

A total of 57 individuals (3\%) of the patients with GD also received an ED diagnosis at any time during the study period ( $8 \%$ of women, $n=39$, and $1 \%$ of men, $n=18, P<$ 0.001 ), among them 14 individuals (1\%) with anorexia-type eating disorder (F50.1-2), 16 (1\%) with bulimia-type disorder (F50.2-3), two (0\%) with other over-eating disorder (F50.4), and 45 (2\%) with other or unspecified eating disorder (F50.8-9).

Table 2. Logistic regression analysis. Variables associated with ED

\begin{tabular}{lccc}
\hline & OR & $\begin{array}{c}95 \% \text { confidence } \\
\text { interval }\end{array}$ & $\begin{array}{c}P \\
\text { value }\end{array}$ \\
\hline Female gender & 7.05 & $3.81-13.04$ & $<0.001$ \\
Drug use disorder & 1.36 & $0.74-2.49$ & 0.32 \\
Bipolar disorder & 1.41 & $0.73-2.73$ & 0.31 \\
Depressive disorder & 2.34 & $1.14-4.80$ & 0.02 \\
Other mood disorder & 1.79 & $0.82-3.95$ & 0.15 \\
Anxiety disorder & 1.58 & $0.72-3.46$ & 0.25 \\
Personality disorder & 1.92 & $1.02-3.63$ & 0.04 \\
Neuropsychiatric & 1.05 & $0.55-1.98$ & 0.89 \\
$\quad$ disorder & & & \\
Age & 0.95 & $0.92-0.97$ & $<0.001$ \\
\hline
\end{tabular}

GD+ED patients were significantly younger $(P=0.005)$ and more likely to be female. Even when controlling for gender, GD + ED was significantly associated with drug use disorders, depressive disorders, bipolar disorders, other mood disorder, anxiety disorders, personality disorders, and neuropsychiatric disorders (Table 1). In logistic regression, controlling all significant variables for one another, ED remained significantly associated with younger age, female gender, depressive disorders, and personality disorders (Table 2).

\section{DISCUSSION}

The current study demonstrates, in a nationwide register sample, that patients with both a GD and an ED during the study period, were more frequently women, younger, and demonstrated a markedly higher psychiatric comorbidity with respect to depression and personality disorders, than GD patients without an ED.

Several studies have shown that impulse-related disorders, such as problematic gambling and compulsive buying, aree associated with greater ED severity and maladaptive personality traits (Alvarez-Moya et al., 2007; FernandezAranda et al., 2006; Jimenez-Murcia et al., 2013); both ED and GD may be associated with high levels of harm avoidance and low levels of self-directedness (Alvarez-Moya et al., 2007). Patients with combined GD and FA have been found to have lower levels of self-directedness (Jimenez-Murcia et al., 2017). Thus, for diverse constructs representing a 
disordered eating behavior, these may share characteristics with a problematic gambling pattern.

Our results show that patients with GD+ED have higher prevalence of personality disorder (PD). As many around $30 \%$ of patients with ED have a diagnosable PD (Godt, 2008), and in a review from Dowling et al. (2015) the prevalence for any PD is $47.9 \%$ among treatment-seeking problem gamblers. The well-known pathway explanatory model for gambling suggests a subgroup of gamblers with early onset, severe psychopathology, high levels of impulsivity and high rates of anti-social personality disorder (Blaszczynski \& Nower, 2002). Thus, personality disorders are prevalent both in $\mathrm{ED}$ and GD, and the fact that PD was even higher in individuals with combined GD+ED may not be surprising. Future studies, involving a larger number of patients and greater statistical power, should attempt to shed further light on temporal associations between GD, ED and PD.

In the Jimenez-Murcia et al. (2017) study, co-occurrence of FA in treatment-seeking GD patients was associated with worse emotional and psychological states, and differences in temperament. In Kim, von Ranson, Hodgins, McGrath, and Tavares (2018) study, individuals with a comorbid binge/ purge type eating disorder reported more days gambling. They had more gambling-related cognitive distortions, impulsivity, suicidality, and other current psychiatric comorbidities including other addictive behaviors. In a study with BED patients (Yip et al., 2011), individuals with comorbid gambling problems had lower self-esteem, higher BMI and more substance use. This further points to a more severe clinical picture in patients with both problem gambling and problematic eating, again in line with the findings of the present study.

Depression also was associated with an ED in the previous study, when controlling for gender and other diagnostic groups. Depression is known to be common ED and $\mathrm{GD}$; in a population of individuals with $\mathrm{ED}$, major depressive disorder was seen in $39 \%$ of AN patients, $50 \%$ of BN patients, and $32 \%$ of BEED patients (Hudson, Hiripi, Pope, \& Kessler, 2007). In the meta-analysis describing comorbidities in GD (Lorains, Cowlishaw, \& Thomas, 2011), major depression occurred in 23\%. Depressive symptoms may often develop before gambling problems (Kennedy et al., 2010), and a subgroup of gamblers may gamble to relieve symptoms of depression and anxiety (Blaszczynski \& Nower, 2002). Another perspective is that mood disorders are likely to be secondary symptoms of financial losses in GD (Kim, Grant, Eckert, Faris, \& Hartman, 2006). Thus, again, given the high prevalence of depression in both GD and ED, separately, it may not seem surprising that the occurrence is even higher in co-morbid GD+ED patients.

The present study may have implications for the clinical assessment and follow-up in problem gamblers, especially women. The study may raise awareness about the rationale for actively screening in patients with either GD or ED, in order to identify the other conditions along with other potential comorbidities (Jimenez-Murcia et al., 2013). Also, based on the present findings, the identification of an ED in a sample of GD patients should lead to further clinical assessments of the higher prevalence of other comorbidities seen here in the combined GD+ED group, and which may or may not cause an even more pronounced clinical problem than the GD or ED themselves. In the treatment of GD, one should be particularly observant about whether there is a parallel ED present, as it appears to be associated with particularly high psychiatric treatment needs. This is of particular importance to highlight in settings where a large part of GD treatment is given outside psychiatry and outside established institutions, and where screening for disordered eating may not typically occur. Our study indicates that the subgroup suffering from the combined condition should be treated where mental illness can be detected and treated, rather than in non-professional treatment such as for example voluntary organizations. Also, ED are more common in women and GD is more common in men. This may limit the awareness of comorbidity between these conditions, and, again, may decrease the likelihood that a patient presenting with one of the conditions is structurally screened for the other. Furthermore, in the line of precision medicine, it is important to be able to identify differentiated phenotypes (and endophenotypes) of patients in order to be able to apply the most indicated treatments in each case, 'tailored' treatments, in order to achieve the best result. Thus, there may be a need for systematic routines for screening tools when meeting these patients, in order to ensure a more tailored approach (Kan, Cardi, Stahl, \& Treasure, 2019).

\section{Limitations}

A registry-based has the limitation of not providing more indepth medical records. The cross-sectional retrospective cohort design does not allow studies of temporality of the variables. Also, in the comparison of the GD+ED subgroup and other GD patients, the obviously uneven distribution between the two groups presents a challenge to the statistical methods, as compared to a study where two patient groups of equal size are compared. Although register data of a diagnosis is unlikely to reflect a date close to the onset of a disorder, future research should include larger samples where temporal association can be assessed. Also, in a study based on a diagnostic records, less can be known about more sub-clinical levels of gambling or eating problems.

\section{CONCLUSIONS}

Our findings suggest clinically relevant differences between GD patients with and without ED. Specifically, we found a greater severity of problems in the comorbid group, including higher likelihood of depression and personality disorder. Unrecognized and untreated ED is likely to be associated with a particularly severe clinical picture in GD, potentially affecting prognosis in this condition. Further research using longitudinal and patient-specific methods is 
required investigating the onset and pattern of comorbid conditions in GD.

Funding sources: The present study was carried out thanks to different co-authors support from CIBERobn and from the state-owned Swedish gambling operator AB Svenska Spel, respectively. The CIBERobn is an initiative of Instituto de Salud Carlos III (ISCIII), Spain.

Authors' contribution: Study concept and design: LL, FFA, SJM, AH. Analysis and interpretation of data: LL, FFA, SJM, AH. Statistical analysis: AH. Obtained funding: FFA, SJM, AH. Study supervision: AH. All authors had full access to all data in the study and take responsibility for the integrity of the data and the accuracy of the data analysis.

Conflict of interest: Hakansson has a position as professor at Lund University which is sponsored by the state-owned gambling operator, partly constituting a gambling monopoly on parts of the gambling market. Also, he has obtained funding from the research council of the same state-owned gambling operator, as well as from the research council of the Swedish alcohol monopoly. Other researchers report no conflicts of interest.

\section{REFERENCES}

Alvarez-Moya, E. M., Jimenez-Murcia, S., Granero, R., Vallejo, J., Krug, I., Bulik, C. M., \& Fernandez-Aranda, F. (2007). Comparison of personality risk factors in bulimia nervosa and pathological gambling. Comprehensive Psychiatry, 48(5), 452457. https://doi.org/10.1016/j.comppsych.2007.03.008.

Bahji, A., Mazhar, M. N., Hudson, C. C., Nadkarni, P., MacNeil, B. A., \& Hawken, E. (2019). Prevalence of substance use disorder comorbidity among individuals with eating disorders: A systematic review and meta-analysis. Psychiatry Research, 273, 58-66. https://doi.org/10.1016/j.psychres.2019.01.007.

Blaszczynski, A., \& Nower, L. (2002). A pathways model of problem and pathological gambling. Addiction, 97(5), 487-499. https://doi.org/10.1046/j.1360-0443.2002.00015.x.

Calado, F., \& Griffiths, M. D. (2016). Problem gambling worldwide: An update and systematic review of empirical research (20002015). Journal of Behavioral Addictions, 5, 592-613. https://doi. org/10.1556/2006.5.2016.073.

Davis, C., Curtis, C., Levitan, R. D., Carter, J. C., Kaplan, A. S., \& Kennedy, J. L. (2011). Evidence that "food addiction" is a valid phenotype of obesity. Appetite, 57(3), 711-717. https://doi.org/ 10.1016/j.appet.2011.08.017.

DeJong, H., Oldershaw, A., Sternheim, L., Samarawickrema, N., Kenyon, M. D., Broadbent, H., ... Schmidt, U. (2013). Quality of life in anorexia nervosa, bulimia nervosa and eating disorder not-otherwise-specified. Journal of Eating Disorders, 1, 43. https://doi.org/10.1186/2050-2974-1-43.

Dowling, N. A., Cowlishaw, S., Jackson, A. C., Merkouris, S. S., Francis, K. L., \& Christensen, D. R. (2015). Prevalence of psychiatric co-morbidity in treatment-seeking problem gamblers: A systematic review and meta-analysis. The Australian and New Zealand Journal of Psychiatry, 49(6), 519-539. https:// doi.org/10.1177/0004867415575774.

Farstad, S. M., von Ranson, K. M., Hodgins, D. C., El-Guebaly, N., Casey, D. M., \& Schopflocher, D. P. (2015). The influence of impulsiveness on binge eating and problem gambling: A prospective study of gender differences in Canadian adults. Psychology of Addictive Behaviors, 29(3), 805-812. https://doi.org/ 10.1037/adb0000069.

Fernandez-Aranda, F., Jimenez-Murcia, S., Alvarez-Moya, E. M., Granero, R., Vallejo, J., \& Bulik, C. M. (2006). Impulse control disorders in eating disorders: Clinical and therapeutic implications. Comprehensive Psychiatry, 47(6), 482-488. https://doi. org/10.1016/j.comppsych.2006.03.002.

Gearhardt, A. N., Corbin, W. R., \& Brownell, K. D. (2009). Food addiction: An examination of the diagnostic criteria for dependence. Journal of Addiction Medicine, 3(1), 1-7. https:// doi.org/10.1097/ADM.0b013e318193c993.

Godt, K. (2008). Personality disorders in 545 patients with eating disorders. European Eating Disorders Review, 16(2), 94-99. https://doi.org/10.1002/erv.844.

Hudson, J. I., Hiripi, E., Pope, H. G. J., \& Kessler, R. C. (2007). The prevalence and correlates of eating disorders in the National Comorbidity Survey Replication. Biological Psychiatry, 61(3), 348-358. https://doi.org/10.1016/j.biopsych.2006.03.040.

Jiménez-Murcia, S., Agüera, Z., Paslakis, G., Munguia, L, Granero, R, Sánchez-González, J, \& Fernández-Aranda, F. (2019). Food addiction in eating disorders and obesity: Analysis of clusters and implications for treatment. Nutrients, 11(11), 2633. https:// doi.org/10.3390/nu11112633.

Jimenez-Murcia, S., Granero, R., Wolz, I., Bano, M., Mestre-Bach, G., Steward, T., ... Fernandez-Aranda, F. (2017). Food addiction in gambling disorder: Frequency and clinical outcomes. Frontiers in Psychology, 8, 473. https://doi.org/10.3389/fpsyg. 2017.00473.

Jimenez-Murcia, S., Steiger, H., Israel, M., Granero, R., Prat, R., Santamaria, J. J., ... Fernandez-Aranda, F. (2013). Pathological gambling in eating disorders: Prevalence and clinical implications. Comprehensive Psychiatry, 54(7), 1053-1060. https://doi. org/10.1016/j.comppsych.2013.04.014.

Kan, C., Cardi, V., Stahl, D., \& Treasure, J. (2019). Precision psychiatry-What it means for eating disorders?. European Eating Disorders Review, 27(1), 3-7. https://doi.org/10.1002/erv.2651.

Karlsson, A., \& Håkansson, A. (2018). Gambling disorder, increased mortality, suicidality, and associated comorbidity: A longitudinal nationwide register study. Journal of Behavioral Addictions, 7(4), 1091-1099. https://doi.org/10.1556/2006.7. 2018.112.

Kennedy, S. H., Welsh, B. R., Fulton, K., Soczynska, J. K., McIntyre, R. S., O’Donovan, C., ... Martin, N. (2010). Frequency and correlates of gambling problems in outpatients with major depressive disorder and bipolar disorder. Canadian Journal of Psychiatry, 55(9), 568-576. https://doi.org/10.1177/ 070674371005500905.

Kim, S. W., Grant, J. E., Eckert, E. D., Faris, P. L., \& Hartman, B. K. (2006). Pathological gambling and mood disorders: Clinical associations and treatment implications. Journal of Affective 
Disorders, 92(1), 109-116. https://doi.org/10.1016/j.jad.2005.12. 040.

Kim, H. S., von Ranson, K. M., Hodgins, D. C., McGrath, D. S., \& Tavares, H. (2018). Demographic, psychiatric, and personality correlates of adults seeking treatment for disordered gambling with a comorbid binge/purge type eating disorder. European Eating Disorders Review, 26(5), 508-518. https://doi.org/10. 1002/erv.2606.

Krug, I., Treasure, J., Anderluh, M., Bellodi, L., Cellini, E., di Bernardo, M., ... Fernandez-Aranda, F. (2008). Present and lifetime comorbidity of tobacco, alcohol and drug use in eating disorders: A European multicenter study. Drug and Alcohol Dependence, 97(1-2), 169-179. https://doi.org/10.1016/j. drugalcdep.2008.04.015.

Langham, E., Thorne, H., Browne, M., Donaldson, P., Rose, J., \& Rockloff, M. (2016). Understanding gambling related harm: A proposed definition, conceptual framework, and taxonomy of harms. BMC Public Health, 16, 80. https://doi.org/10.1186/ s12889-016-2747-0.

Lorains, F. K., Cowlishaw, S., \& Thomas, S. A. (2011). Prevalence of comorbid disorders in problem and pathological gambling: Systematic review and meta-analysis of population surveys. Addiction, 106(3), 490-498. https://doi.org/10.1111/j.13600443.2010.03300.x.
Pursey, K. M., Stanwell, P., Gearhardt, A. N., Collins, C. E., \& Burrows, T. L. (2014). The prevalence of food addiction as assessed by the Yale food addiction scale: A systematic review. Nutrients, 6(10), 4552-4590. https://doi.org/10.3390/ nu6104552.

Qian, J., Hu, Q., Wan, Y., Li, T., Wu, M., Ren, Z., \& Yu, D. (2013). Prevalence of eating disorders in the general population: A systematic review. Shanghai Archives of Psychiatry, 25(4), 212223. https://doi.org/10.3969/j.issn.1002-0829.2013.04.003.

Romero, X., Agüera, Z., Granero, R., Sánchez, I, Riesco, N, JiménezMurcia, S, \& Fernández-Aranda, F. (2019). Is food addiction a predictor of treatment outcome among patients with eating disorder? European Eating Disorders Review, 27(6), 700-711. https://doi.org/10.1002/erv.2705.

von Ranson K. M., Wallace L. M., Holub A., \& Hodgins D. C. (2013). Eating disorders, substance use disorders, and impulsiveness among disordered gamblers in a community sample. European Eating Disorders Review, 21(2), 148-154. https://doi. org/10.1002/erv.2207.

Yip, S. W., White, M. A., Grilo, C. M., \& Potenza, M. N. (2011). An exploratory study of clinical measures associated with subsyndromal pathological gambling in patients with binge eating disorder. Journal of Gambling Studies, 27(2), 257-270. https:// doi.org/10.1007/s10899-010-9207-z. 